\title{
Multicomponent Reaction, Ionic Liquid and Aqua Mediated: A Perfect Synergy for Eco-compatible Heterocyclic Synthesis
}

\author{
FAIZA BOUKEZZOULA, TAOUES BOUMOUD*, \\ BOUDJEMAA BOUMOUD and ABDELMADJID DEBACHE
}

Laboratoire de Synthèse de Molécules d'Intérêts Biologiques, Département de Chimie, Faculté des Sciences Exactes, Université Constantine 1, 25000 Constantine, Algérie.

boumoudt@yahoo.com

Received 30 November 2014 / Accepted 16 December 2014

\begin{abstract}
The combination of multicomponent reactions as potential synthetic processes with ionic liquids as catalysts and water as reaction medium is a suitable approach that respects, in some extent, the requirements of sustainable chemistry. In this context, we describe herein an efficient and high yielding four-component synthesis of 6-amino-4-alkyl/aryl-3-methyl-2,4-dihydropyrano[2,3c]pyrazole-5-carbonitriles involving ethyl acetoacetate, hydrazine hydrate, malononitrile, and various aldehydes using methyltriphenylphosphonium bromide as calalyst and water as solvent under mild reaction conditions.
\end{abstract}

Keywords: Pyranopyrazoles, Triphenylphosphine, Methyltriphenylphosphonium bromide, Ionic liquid, Aqueous medium, Multicomponent reactions, Organocatalyst, Rate enhancements

\section{Introduction}

Nowadays, the challenge in synthesis is how a molecule can be prepared from readily available starting materials, in maximum yield and high atom and step economy via a clean and environmentally benign chemical process. In this sense, multi-component reactions (MCRs); known to be selective, effective atom economic, time saving and easy to perform; have attracted significant interest from pharmaceutical groups ${ }^{1}$.

Due to their environmentally benign nature, high polarity and good thermal stability, ionic liquids (ILs) have received substantial attention in organic synthesis in the past decade. Furthermore, high yields, shorter reaction times, cleaner reaction products and high selectivity are often obtained on using ILs either as solvents or as catalysts ${ }^{2}$ as a result; recently, ionic liquids have attracted increasing interest in the context of green organic synthesis.

Besides ILs, carrying organic reactions in aqueous media is another attractive area in green chemistry ${ }^{3}$. As a reaction medium, water offers several advantages such as environmental compatibility, reduction of by-products and direct isolation of products by 
precipitation and filtration as they are often insoluble. Furthermore, considerable rate enhancements are often observed in reactions carried in water over those in organic solvents ${ }^{4}$, in particular, reactions with negative activation volume ${ }^{5}$ such as multicomponent reactions ${ }^{6}$.

Pyranopyrazoles constitute important fused heterocyclic compounds; thus their synthesis has received much attention especially in the field of medicinal/pharmaceutical chemistry due to their wide range of biological/pharmacological activities such as anticancer, antimicrobial, anti-inflammatory, insecticidal and as biodegradable agrochemicals ${ }^{7}$. The first procedure to synthesize pyranopyrazole derivatives involves the reaction between 3-methyl-1-phenylpyrazolin-5-one and tetracyanoethylene under basic conditions $^{8}$. Owing to the above mentioned properties, various methods were developed for the synthesis of these compounds among which we cite the two-component reaction involving pyran derivatives and hydrazine hydrate under combined microwave and ultrasound irradiation ${ }^{9}$, the three-component reaction between pyrazolone, an aldehyde and malononitrile using triethylamine as catalyst ${ }^{10}$, the four-component synthesis between ethyl acetoacetate, hydrazine hydrate, aldehyde, and malononitrile in the presence of bases such piperdine, pyrrolidine, morpholine and triethylamine as catalysts ${ }^{11}$. Recently, several catalysts have been reported for the one-pot synthesis of pyranopyrazole derivatives including glycine ${ }^{12}, L$-proline and $\gamma$-alumina ${ }^{13}$, per-6-amino- $\beta$-cyclodextrin ${ }^{14}$ andcetyltrimethylammonium chloride $(\mathrm{CTACl})^{15}$.

Taking into account the importance of pyranopyrazoles, the significant rate enhancement of MCRs in water and the merits of organocatalysis induced by ILs, herein, we wish to report the catalytic efficiency of methyltriphenylphosphonium bromide (MTPB) for the synthesis of 6-amino-5-cyano-4-aryl-4H-pyrazolo[3,4-b]pyran derivatives 5 via the four-component reaction of hydrazine hydrate $\mathbf{1}$, ethyl acetoacetate 2 , aromatic aldehydes $\mathbf{3}$ and malononitrile $\mathbf{4}$ in aqueous medium (Scheme 1).

\section{Experimental}

All chemicals were used without further purification. Reactions were monitored by thinlayer chromatography (TLC), visualising with ultraviolet. Melting points were determined on Banc Kofler apparatus and are uncorrected. IR spectra were recorded on Chimadzu FT IR $8201 \mathrm{PC}$ spectrometer as $\mathrm{KBr}$ pellets with absorptions in $\mathrm{cm}^{-1}$. ${ }^{1} \mathrm{H}$ NMR and ${ }^{13} \mathrm{C} \mathrm{NMR}$ spectra were recorded on a Bruker BRUKER AVANCE DPX spectrometer using DMSO-d $\mathrm{d}_{6}$ as solvent and TMS as an internal standard. Chemical shifts $(\delta)$ are expressed in ppm, downfield from internal standard TMS and $J$ values in hertz $(\mathrm{Hz})$.

General procedure for the preparation of 6-Amino-4-aryl-3-methyl-2,4dihydropyrano[2,3-c]pyrazole-5-carbonitriles or carboxylate (5a-m)

A mixture of hydrazine hydrate $1(0.107 \mathrm{~g}, 2.0 \mathrm{mmol})$, ethyl acetoacetate $2(0.260 \mathrm{~g}$, $2.0 \mathrm{mmol})$, aromatic aldehyde derivatives $3(2.0 \mathrm{mmol})$ and malononitrile $4(0.132 \mathrm{~g}$, $2.0 \mathrm{mmol})$ in water $(5 \mathrm{~mL})$ was refluxed under stirring for $1 \mathrm{~h}$ in the presence of MTPB $(5 \mathrm{~mol} \%)$. The precipitated solid was filtered, washed with water and then with a mixture of ethyl acetate/hexane (20/80). The product obtained was pure by TLC and ${ }^{1} \mathrm{H}$ NMR spectroscopy. However, the products were further purified by recrystallization from ethanol. All the synthesized compounds are reported in Table 4 and were confirmed by their physical constants and characterized by IR, ${ }^{1} \mathrm{H}$ and ${ }^{13} \mathrm{C}$ NMR. The spectroscopic data were in full agreement with the literature values. 


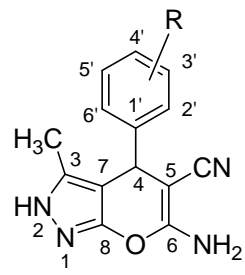

\section{Spectral data for prepared compounds}

6-Amino-3-methyl-4-phenyl-2,4-dihydropyrano [2, 3-c] pyrazole-5-carbonitrile (5a) White crystals, mp. $245-246{ }^{\circ} \mathrm{C} ; \mathrm{V}_{\max }(\mathrm{KBr}): 3309,3170\left(\mathrm{NH}_{2}\right), 2191(\mathrm{CN}), 1647(\mathrm{C}=\mathrm{N})$, $1610,1604(\mathrm{Ar}) \mathrm{cm}^{-1} ;{ }^{1} \mathrm{H}$ NMR(250 MHz, DMSO-d 6 ) $\delta 12.02(\mathrm{~s}, 1 \mathrm{H}, \mathrm{NH}), 7.45-7.15(\mathrm{~m}$, $5 \mathrm{H}$, arom), $6.74\left(\mathrm{~s}, 2 \mathrm{H}, \mathrm{NH}_{2}\right), 4.56(\mathrm{~s}, 1 \mathrm{H}, 4-\mathrm{H}), 1.79\left(\mathrm{~s}, 3 \mathrm{H}, \mathrm{CH}_{3}\right) ;{ }^{13} \mathrm{C}$ NMR $(63.9 \mathrm{MHz}$, DMSO-d $_{6}$ ) $\delta 159.3(\mathrm{C} 6), 153.2(\mathrm{C} 3), 142.2$ (C8), 133.9 (C1'), $126.6\left(\mathrm{C}^{\prime}, \mathrm{C} 6{ }^{\prime}\right), 125.8$ (C3',C5') 125.0 (C4'), $119.1(\mathrm{CN}), 95.9$ (C7), 55.8 (C5), 34.8 (C4), 8.1( $\left.\mathrm{CH}_{3}\right)$.

6-Amino-4-(4-chlorophenyl)-3-methyl-2,4-dihydropyrano[2,3-c]pyrazole-5carbonitrile (5b)

Yellow solid, mp. $239-240{ }^{\circ} \mathrm{C} ; \mathrm{V}_{\max }(\mathrm{KBr}): 3367,3174\left(\mathrm{NH}_{2}\right), 2187(\mathrm{CN}), 1653(\mathrm{C}=\mathrm{N}), 1600$ (Ar) $\mathrm{cm}^{-1} ;{ }^{1} \mathrm{H}$ NMR $\left(250 \mathrm{MHz}, \mathrm{DMSO}_{-} \mathrm{d}_{6}\right): \delta 12.0(\mathrm{~s}, 1 \mathrm{H}, \mathrm{NH}), 7.17\left(\mathrm{~d}, 2 \mathrm{H}, J=7.0, \mathrm{H}_{\text {arom }}\right.$ ), $7.07\left(\mathrm{~d}, J=7.0 \mathrm{~Hz}, 2 \mathrm{H}, \mathrm{H}_{\text {arom }}\right), 6.38\left(\mathrm{~s}, 2 \mathrm{H}, \mathrm{NH}_{2}\right), 4.51(\mathrm{~s}, 1 \mathrm{H}, 4-\mathrm{H}), 1.77\left(\mathrm{~s}, 3 \mathrm{H}, \mathrm{CH}_{3}\right) ;{ }^{13} \mathrm{C}$ NMR (63.9 MHz, DMSO-d $\left.{ }_{6}\right) \delta 159.8$ (C6), 153.8 (C3), 141.6 (C8), 134.8(C4'), 130.8(C1'), 128.0 (C2',C6'), 127.2 (C3',5'), $119.7(\mathrm{CN}), 95.9$ (C7), $56.6(\mathrm{C} 5), 35.0(\mathrm{C} 4), 8.9\left(\mathrm{CH}_{3}\right)$.

6-Amino-4-(4-hydroxyphenyl)-3-methyl-2,4-dihydropyrano[2,3-c]pyrazole-5carbonitrile (5c)

Yellow solid, mp. $229-230{ }^{\circ} \mathrm{C} ; \mathrm{V}_{\max }(\mathrm{KBr}): 3300,3130\left(\mathrm{NH}_{2}\right), 2179(\mathrm{CN}), 1650(\mathrm{C}=\mathrm{N}), 1596$ (Ar) $\mathrm{cm}^{-1} ;{ }^{1} \mathrm{H}$ NMR $\left(250 \mathrm{MHz}, \mathrm{DMSO}-\mathrm{d}_{6}\right) \delta 12.0(\mathrm{~s}, 1 \mathrm{H}, \mathrm{NH}), 9.21(\mathrm{~s}, 1 \mathrm{H}, \mathrm{OH}), 7.0(\mathrm{~d}, J$ $=7.0 \mathrm{~Hz}, 2 \mathrm{H}$, arom $), 6.70(\mathrm{~d}, J=6.7 \mathrm{~Hz}, 2 \mathrm{H}$, arom $), 6.65\left(\mathrm{~s}, 2 \mathrm{H}, \mathrm{NH}_{2}\right), 4.44(\mathrm{~s}, 1 \mathrm{H}, 4-\mathrm{H}), 1.79$ $\left(\mathrm{s}, 3 \mathrm{H}, \mathrm{CH}_{3}\right) ;{ }^{13} \mathrm{C}$ NMR (63.9 MHz, DMSO-d 6 ) $\delta 160.6$ (C6), 154.8 (C3), 135.5 (C8), 156.0, 134.6, 128.4 and $115.1\left(\mathrm{C}_{\text {arom }}\right), 121.0(\mathrm{CN}), 98.0(\mathrm{C} 7), 58.0(\mathrm{C} 5), 35.6(\mathrm{C} 4), 9.8\left(\mathrm{CH}_{3}\right)$.

6-Amino-4-(4-methoxyphenyl)-3-methyl-2,4-dihydropyrano[2,3-c]pyrazole-5carbonitrile (5d)

White solid, mp.225-226 ${ }^{\circ} \mathrm{C} ; \mathrm{V}_{\max }(\mathrm{KBr}): 3360\left(\mathrm{NH}_{2}\right), 2191(\mathrm{CN}), 1640(\mathrm{C}=\mathrm{N}), 1600(\mathrm{Ar}) \mathrm{cm}^{-}$ ${ }^{1}$; ${ }^{1} \mathrm{H}$ NMR $\left(250 \mathrm{MHz}, \mathrm{DMSO}-\mathrm{d}_{6}\right) \delta 12.0(\mathrm{~s}, 1 \mathrm{H}, \mathrm{NH}), 7.03(\mathrm{~d}, J=8.6 \mathrm{~Hz}, 2 \mathrm{H}$, arom $), 6.70(\mathrm{~d}, J$ $=8.6 \mathrm{~Hz}, 2 \mathrm{H}$, arom $), 6.60\left(\mathrm{~s}, 2 \mathrm{H}, \mathrm{NH}_{2}\right), 4.45(\mathrm{~s}, 1 \mathrm{H}, 4-\mathrm{H}), 3.68\left(\mathrm{~s}, 3 \mathrm{H}, \mathrm{OCH}_{3}\right), 1.79(\mathrm{~s}, 3 \mathrm{H}$, $\left.\mathrm{CH}_{3}\right) ;{ }^{13} \mathrm{C}$ NMR (63.9 MHz, DMSO-d ${ }_{6}$ ) $\delta 161.7$ (C6), $154.8(\mathrm{C} 3), 136.3(\mathrm{C} 8), 158.0,135.6$, 128.5 and $113.6\left(\mathrm{C}_{\text {arom }}\right), 120.9(\mathrm{CN}), 97.7(\mathrm{C} 7), 57.9(\mathrm{C} 5), 54.9\left(\mathrm{OCH}_{3}\right), 35.7(\mathrm{C} 4), 9.8\left(\mathrm{CH}_{3}\right)$.

6-Amino-3-methyl-4-(4-methylphenyl)-2,4-dihydropyrano[2,3-c]pyrazole-5carbonitrile (5e)

Yellow crystals, mp.219-220 ${ }^{\circ} \mathrm{C} ; \mathrm{V}_{\max }(\mathrm{KBr}): 3400,3047\left(\mathrm{NH}_{2}\right), 2191(\mathrm{CN}), 1643(\mathrm{C}=\mathrm{N})$, $1600(\mathrm{Ar}) \mathrm{cm}^{-1} ;{ }^{1} \mathrm{H}$ NMR $\left(250 \mathrm{MHz}, \mathrm{DMSO}_{-}\right) \delta 12.11(\mathrm{~s}, 1 \mathrm{H}, \mathrm{NH}), 7.12(\mathrm{~d}, J=7.0 \mathrm{~Hz}$, $2 \mathrm{H}$, arom), 7.05 (d, $J=7.0 \mathrm{~Hz}, 2 \mathrm{H}$, arom), 6.89(s, 2H, $\left.\mathrm{NH}_{2}\right), 4.55(\mathrm{~s}, 1 \mathrm{H}, 4-\mathrm{H}), 2.27(\mathrm{~s}, 3 \mathrm{H}$, $\left.\mathrm{CH}_{3}\right) 1.79\left(\mathrm{~s}, 3 \mathrm{H}, \mathrm{CH}_{3}\right) ;{ }^{13} \mathrm{C}$ NMR $\left(63.9 \mathrm{MHz}, \mathrm{DMSO}_{6}\right) \delta 160.8(\mathrm{C} 6), 154.8(\mathrm{C} 3), 135.8$ (C8), 141.6, 129.1, 127.5 and $120.9\left(\mathrm{C}_{\text {arom }}\right), 119.4(\mathrm{CN}), 97.8(\mathrm{C} 7), 57.4(\mathrm{C} 5), 35.9(\mathrm{C} 4)$, $20.7\left(\mathrm{CH}_{3}\right), 9.9\left(\mathrm{CH}_{3}\right)$. 
6-Amino-4-(4-nitrophenyl)-3-methyl-2,4-dihydropyrano[2,3-c]pyrazole-5carbonitrile (5f)

Brown crystals, mp. $249-250{ }^{\circ} \mathrm{C} ; \mathrm{V}_{\max }(\mathrm{KBr}): 3475,3186\left(\mathrm{NH}_{2}\right), 2190(\mathrm{CN}), 1651(\mathrm{C}=\mathrm{N})$, $1601(\mathrm{Ar}) \mathrm{cm}^{-1} ;{ }^{1} \mathrm{H}$ NMR $\left(250 \mathrm{MHz}, \mathrm{DMSO}-\mathrm{d}_{6}\right) \delta 12.00(\mathrm{~s}, 1 \mathrm{H}, \mathrm{NH}), 8.0(\mathrm{~d}, J=8.6 \mathrm{~Hz}, 2 \mathrm{H}$, arom), $7.4\left(\mathrm{~d}, J=8.6 \mathrm{~Hz}, 2 \mathrm{H}\right.$, arom), $6.6\left(\mathrm{~s}, 2 \mathrm{H}, \mathrm{NH}_{2}\right), 4.70(\mathrm{~s}, 1 \mathrm{H}, 4-\mathrm{H}), 1.8\left(\mathrm{~s}, 3 \mathrm{H}, \mathrm{CH}_{3}\right)$; ${ }^{13} \mathrm{C}$ NMR (63.9 MHz, DMSO-d 6 ) $\delta 159.8$ (C6), 150.3 (C3), 134.5 (C8), 145.1, 153.4, 127.3 and $122.3\left(\mathrm{C}_{\text {arom }}\right), 119.1(\mathrm{CN}), 94.9(\mathrm{C} 7), 55.1(\mathrm{C} 5), 35.0(\mathrm{C} 4), 8.5\left(\mathrm{CH}_{3}\right)$.

6-Amino-4-(3-hydroxyphenyl)-3-methyl-2,4-dihydropyrano[2,3-c]pyrazole-5carbonitrile (5g)

White powder, mp.248-249 ${ }^{\circ} \mathrm{C} ; \mathrm{V}_{\max }(\mathrm{KBr}): 3363,3166\left(\mathrm{NH}_{2}\right), 2179(\mathrm{CN}), 1647(\mathrm{C}=\mathrm{N}), 1600$ ( Ar) $\mathrm{cm}^{-1}$; ${ }^{1} \mathrm{H}$ NMR $\left(250 \mathrm{MHz}, \mathrm{DMSO}_{6}\right) \delta 11.91(\mathrm{~s}, 1 \mathrm{H}, \mathrm{NH}), 9.12(\mathrm{~s}, 1 \mathrm{H}, \mathrm{OH}), 7.05(\mathrm{td}, J$ $=7.7 \mathrm{~Hz}, 1 \mathrm{H}$, arom $), 6.70\left(\mathrm{~s}, 2 \mathrm{H}, \mathrm{NH}_{2}\right), 6.60(\mathrm{~m}, 3 \mathrm{H}, \operatorname{arom}), 4.40(\mathrm{~s}, 1 \mathrm{H}, 4-\mathrm{H}), 1.82(\mathrm{~s}, 3 \mathrm{H}$, $\left.\mathrm{CH}_{3}\right) ;{ }^{13} \mathrm{C}$ NMR (63.9 MHz, DMSO-d 6 ) $\delta 161.0$ (C6), 154.8 (C3), $135.5(\mathrm{C} 8), 157.4,145.7$, 129.1, 120.8, 114.2 and $113.8\left(\mathrm{C}_{\text {arom }}\right), 118.1(\mathrm{CN}), 97.5$ (C7), 57.5 (C5), $36.3(\mathrm{C} 4), 9.8\left(\mathrm{CH}_{3}\right)$.

6-Amino-4-(3-nitrophenyl)-3-methyl-2,4-dihydropyrano[2,3-c]pyrazole-5carbonitrile (5h)

Brown powder, mp. 229-230 ${ }^{\circ} \mathrm{C} ; \mathrm{V}_{\max }(\mathrm{KBr}): 3489,3191\left(\mathrm{NH}_{2}\right), 2193(\mathrm{CN}), 1648(\mathrm{C}=\mathrm{N})$, $1600(\mathrm{Ar}) \mathrm{cm}^{-1} ;{ }^{1} \mathrm{H}$ NMR $\left(250 \mathrm{MHz}, \mathrm{DMSO}_{-} \mathrm{d}_{6}\right) \delta 12.02(\mathrm{~s}, 1 \mathrm{H}, \mathrm{NH}), 8.0(\mathrm{~m}, 2 \mathrm{H}$, arom $), 7.6$ (m, $2 \mathrm{H}$, arom), $6.6\left(\mathrm{~s}, 2 \mathrm{H}, \mathrm{NH}_{2}\right), 4.71(\mathrm{~s}, 1 \mathrm{H}, 4-\mathrm{H}), 1.8\left(\mathrm{~s}, 3 \mathrm{H}, \mathrm{CH}_{3}\right) ;{ }^{13} \mathrm{C} \mathrm{NMR}(63.9 \mathrm{MHz}$, DMSO-d $\left.{ }_{6}\right) \delta 156.0(\mathrm{C} 6), 146.8(\mathrm{C} 3), 134.8(\mathrm{C} 8), 153.7,145.2,132.9,128.5,120.9$ and 120.7 ( $\left.\mathrm{C}_{\text {arom }}\right), 119.3(\mathrm{CN}), 95.2(\mathrm{C} 7), 55.7$ (C5), $35.1(\mathrm{C} 4), 8.7\left(\mathrm{CH}_{3}\right)$.

6-Amino-4-(2-methoxyphenyl)-3-methyl-2,4-dihydropyrano[2,3-c]pyrazole-5carbonitrile (5i)

White powder, mp.253-254 ${ }^{\circ} \mathrm{C} ; \mathrm{V}_{\max }(\mathrm{KBr}): 3336\left(\mathrm{NH}_{2}\right), 2191(\mathrm{CN}), 1654(\mathrm{C}=\mathrm{N}), 1600(\mathrm{Ar}) \mathrm{cm}^{-1}$; ${ }^{1} \mathrm{H}$ NMR $\left(250 \mathrm{MHz}, \mathrm{DMSO}-\mathrm{d}_{6}\right) \delta 12.00(\mathrm{~s}, 1 \mathrm{H}, \mathrm{NH}), 7.17(\mathrm{td}, J=7.6 \mathrm{~Hz}, 1 \mathrm{H}$, arom $), 6.92(\mathrm{~m}$, $3 \mathrm{H}$, arom $), 6.72\left(\mathrm{~s}, 2 \mathrm{H}, \mathrm{NH}_{2}\right), 5.00(\mathrm{~s}, 1 \mathrm{H}, 4-\mathrm{H}), 3.80\left(\mathrm{~s}, 3 \mathrm{H}, \mathrm{OCH}_{3}\right), 1.80\left(\mathrm{~s}, 3 \mathrm{H}, \mathrm{CH}_{3}\right) ;{ }^{13} \mathrm{C} \mathrm{NMR}$ (63.9 MHz, DMSO-d ${ }_{6}$ ) $\delta 161.5$ (C6), 155.0 (C3), 135.0 (C8), 156.2, 132.1, 128.6, 127.7, 120.9 and $110.9\left(\mathrm{C}_{\text {arom }}\right), 120.7(\mathrm{CN}), 97.7(\mathrm{C} 7), 56.4(\mathrm{C} 5), 55.4\left(\mathrm{OCH}_{3}\right), 28.9(\mathrm{C} 4), 9.5\left(\mathrm{CH}_{3}\right)$.

6-Amino-4-(2-nitrophenyl)-3-methyl-2, 4-dihydropyrano [2, 3-c] pyrazole-5carbonitrile (5j)

Blackishpowder,mp. 246-247 ${ }^{\circ} \mathrm{C} ; \mathrm{V}_{\max }(\mathrm{KBr}): 3477,3170\left(\mathrm{NH}_{2}\right), 2188(\mathrm{CN}), 1650(\mathrm{C}=\mathrm{N})$, $1600(\mathrm{Ar}) \mathrm{cm}^{-1} ;{ }^{1} \mathrm{H}$ NMR $\left(250 \mathrm{MHz}, \mathrm{DMSO}-\mathrm{d}_{6}\right) \delta 12.00(\mathrm{~s}, 1 \mathrm{H}, \mathrm{NH}), 7.8(\mathrm{~d}, J=8 \mathrm{~Hz}, 1 \mathrm{H}$, arom), 7.6(t, $J=7.5 \mathrm{~Hz}, 1 \mathrm{H}$, arom), 7.4(t, $J=7.6 \mathrm{~Hz}, 1 \mathrm{H}$, arom), 7.3(d, $J=8 \mathrm{~Hz}, 1 \mathrm{H}$, arom), $6.9\left(\mathrm{~s}, 2 \mathrm{H}, \mathrm{NH}_{2}\right), 5.1(\mathrm{~s}, 1 \mathrm{H}, 4-\mathrm{H}), 1.8\left(\mathrm{~s}, 3 \mathrm{H}, \mathrm{CH}_{3}\right) ;{ }^{13} \mathrm{C} \mathrm{NMR}\left(63.9 \mathrm{MHz}, \mathrm{DMSO}_{-}\right) \delta$ 161.1 (C6), 149.0 (C3), 137.8 (C8), 154.9, 135.6, 133.1, 131.1, 127.8 and $123.3\left(\mathrm{C}_{\text {arom }}\right)$, $120.1(\mathrm{CN}), 96.3(\mathrm{C} 7), 56.2(\mathrm{C} 5), 31.1(\mathrm{C} 4), 9.5\left(\mathrm{CH}_{3}\right)$.

6-Amino-4-(4-bromophenyl)-3-methyl-2,4-dihydropyrano[2,3-c]pyrazole-5carbonitrile (5k)

Yellow crystals, mp.180-183 ${ }^{\circ} \mathrm{C} ; \mathrm{V}_{\max }(\mathrm{KBr}): 3394,3182\left(\mathrm{NH}_{2}\right), 2187(\mathrm{CN}), 1650(\mathrm{C}=\mathrm{N})$, 1600 (Ar) $\mathrm{cm}^{-1} ;{ }^{1} \mathrm{H}$ NMR $\left(250 \mathrm{MHz}, \mathrm{DMSO}_{-} \mathrm{d}_{6}\right) \delta 12.15(\mathrm{~s}, 1 \mathrm{H}), 7.41(\mathrm{~d}, J=7.0 \mathrm{~Hz}, 2 \mathrm{H}$, arom), $7.11\left(\mathrm{~d}, J=7.1 \mathrm{~Hz}, 2 \mathrm{H}\right.$, arom), 6.82(s, $\left.2 \mathrm{H}, \mathrm{NH}_{2}\right), 4.57(\mathrm{~s}, 1 \mathrm{H}, 4-\mathrm{H}), 1.78(\mathrm{~s}, 3 \mathrm{H}$, $\left.\mathrm{CH}_{3}\right) ;{ }^{13} \mathrm{C}$ NMR (63.9 MHz, DMSO-d 6 ) $\delta 161.2$ (C6), 154.9 (C3), 143.9 (C8), 136.1, 131.6, 129.9 and $121.0\left(\mathrm{C}_{\text {arom }}\right), 120.2(\mathrm{CN}), 97.3(\mathrm{C} 7), 57.1(\mathrm{C} 5), 36.1(\mathrm{C} 4), 10.0\left(\mathrm{CH}_{3}\right)$. 
6-Amino-3-methyl-4-(2-thienyl)-2,4-dihydropyrano[2,3-c]pyrazole-5-carbonitrile (5l) White solid, mp.226-228 ${ }^{\circ} \mathrm{C}$; $\mathrm{V}_{\max }(\mathrm{KBr}): 3355\left(\mathrm{NH}_{2}\right), 2191(\mathrm{CN}), 1647(\mathrm{C}=\mathrm{N}), 1604(\mathrm{Ar}) \mathrm{cm}^{-1}$; ${ }^{1} \mathrm{H}$ NMR $\left(250 \mathrm{MHz}, \mathrm{DMSO}_{6}\right) \delta 12.13(\mathrm{~s}, 1 \mathrm{H}, \mathrm{NH}), 7.31(\mathrm{~d}, J=3.8 \mathrm{~Hz}, 1 \mathrm{H}$, arom $) 7.00(\mathrm{~d}, J$ $=3.3 \mathrm{~Hz}, 1 \mathrm{H}$, arom), 6.91(td, $J=3.4 \mathrm{~Hz}, 1 \mathrm{H}$, arom), $6.88\left(\mathrm{~s}, 2 \mathrm{H}, \mathrm{NH}_{2}\right), 4.89(\mathrm{~s}, 1 \mathrm{H}, 4-\mathrm{H}), 1.92$ (s, 3H, $\left.\mathrm{CH}_{3}\right) ;{ }^{13} \mathrm{C}$ NMR (63.9 MHz, DMSO-d ${ }_{6}$ ) $\delta 160.6$ (C6), 154.3 (C3), 149.66 (C8), 126.3, 124.7, 124.2 and $120.6\left(\mathrm{C}_{\text {arom }}\right), 97.4(\mathrm{C} 7), 57.5(\mathrm{C} 5), 31.5(\mathrm{C} 4), 9.7\left(\mathrm{CH}_{3}\right)$.

6-Amino-4-(4-(dimethylamino) phenyl)-3-methyl-2, 4-dihydropyrano [2,3-c] pyrazole5-carbonitrile (5m)

Red crystals, mp.219-222 ${ }^{\circ} \mathrm{C} ; \mathrm{V}_{\max }(\mathrm{KBr}): 3390\left(\mathrm{NH}_{2}\right), 2200(\mathrm{CN}), 1643(\mathrm{C}=\mathrm{N}), 1600(\mathrm{Ar})$ $\mathrm{cm}^{-1}$; ${ }^{1} \mathrm{H}$ NMR $\left(250 \mathrm{MHz}, \mathrm{DMSO}-\mathrm{d}_{6}\right) \delta 11.88(\mathrm{~s}, 1 \mathrm{H}), 6.95(\mathrm{~d}, J=8.6 \mathrm{~Hz}, 2 \mathrm{H}), 6.60(\mathrm{~d}, J=$ $8.7 \mathrm{~Hz}, 2 \mathrm{H}), 6.46(\mathrm{~s}, 2 \mathrm{H}), 4.4(\mathrm{~s}, 1 \mathrm{H}), 2.86(\mathrm{~s}, 6 \mathrm{H}), 1.78(\mathrm{~s}, 3 \mathrm{H}) ;{ }^{13} \mathrm{C} \mathrm{NMR}(63.9 \mathrm{MHz}$, DMSO-d $\left.{ }_{6}\right) \delta 158.4(\mathrm{C} 6), 149.0(\mathrm{C} 3), 133.5(\mathrm{C} 8), 154.0,127.9,120.3,112.1\left(\mathrm{C}_{\text {arom }}\right), 118.7$ $(\mathrm{CN}), 97.9(\mathrm{C} 7), 69.5(\mathrm{C} 5), 58.3\left(2 \mathrm{CH}_{3}\right), 35.5(\mathrm{C} 4), 9.7\left(\mathrm{CH}_{3}\right)$.

\section{Results and Discussion}

The reaction (Scheme 1) between hydrazine hydrate, ethyl acetoacetate, malonitrile, and benzaldehyde $\left(\mathrm{R}=\mathrm{C}_{6} \mathrm{H}_{5}\right)$ was chosen as a model condensation reaction for optimizing the various reaction parameters: solvent, temperature, catalyst and concentration.

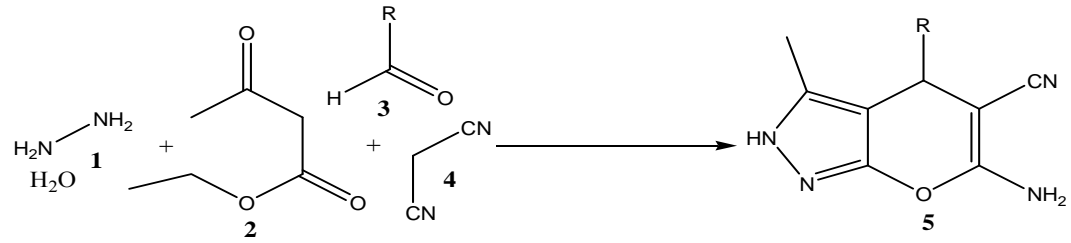

\section{Scheme 1}

Initially, the reaction was tried without any catalyst in solvent-free conditions at ambient temperature, but the reaction could not complete even after $24 \mathrm{~h}$ stirring (Table 1, entry 1). Interestingly, when $5 \mathrm{~mL}$ of water was added to the reaction mixture, an oily product was obtained (Table 1, entry 2) and on raising the temperature of the reaction to $100{ }^{\circ} \mathrm{C}$, the desired product was obtained within $4 \mathrm{~h}$ in $70 \%$ yield (Table 1 , entry 3 ). To increase the reaction rate and minimize the consumption of energy, we performed the model reaction using $5 \mathrm{~mol} \%$ of $\mathrm{PPh}_{3}$ and MTPB as catalysts in aqueous media under reflux conditions. From these preliminary studies, it was observed that (i) the rate of the catalyzed reaction is higher than the corresponding uncatalyzed one at the same temperature and (ii) MTPB is far superior to $\mathrm{PPh}_{3}$ for efficient catalysis (Table 1, entries 4, 5).

Table 1. The Influence of temperature on one-pot condensation of ethyl acetoacetate, hydrazine hydrate, benzaldehyde and malononitrile

\begin{tabular}{cccccc}
\hline Entry & Catalyst & Solvent & Temperature, ${ }^{\circ} \mathrm{C}$ & Time, $\mathrm{h}$ & Yield, \% \\
\hline 1 & Cat free & Neat & Room temperature & 24 & - \\
2 & Cat free & $\mathrm{H}_{2} \mathrm{O}$ & Room temperature & 24 & oil \\
3 & Cat free & $\mathrm{H}_{2} \mathrm{O}$ & Reflux & 4 & 70 \\
4 & $\mathrm{MTPB}(5 \mathrm{~mol} \%)$ & $\mathrm{H}_{2} \mathrm{O}$ & Reflux & $1 \mathrm{~h}$ & 94 \\
5 & $\mathrm{PPh}_{3}(5 \mathrm{~mol} \%)$ & $\mathrm{H}_{2} \mathrm{O}$ & Reflux & $1.5 \mathrm{~h}$ & 76 \\
\hline
\end{tabular}

The choice of a solvent is a crucial factor for multicomponent reactions, so different organic solvents were examined for the reaction (Table 2, entries 2-6) and we found that 
water was the solvent of choice which provided the highest rate and yield (Table 2, entry 6). Similar yield was also obtained under solvent free conditions but relatively longer reaction time was needed (Table 2, entry 1 ).

Table 2. The Influence of solvent on the model reaction in the presence of MTPB (5 mol\%)

\begin{tabular}{lllll}
\hline Entry & Solvent & Temperature & Time, $\mathrm{h}$ & Yield, ${ }^{\mathrm{b}}$ \\
\hline 1 & Solvent-free & $80^{\circ} \mathrm{C}$ & 3 & 91 \\
2 & $\mathrm{CH}_{3} \mathrm{CN}$ & reflux & 5 & 67 \\
3 & $\mathrm{DMSO}$ & reflux & 2 & 55 \\
4 & EtOH & reflux & 5 & 69 \\
5 & EtOH$/ \mathrm{H}_{2} \mathrm{O}$ & reflux & 1 & 84 \\
6 & $\mathrm{H}_{2} \mathrm{O}$ & reflux & 1 & 94 \\
\hline
\end{tabular}

${ }^{a}$ Reaction conditions: ethyl acetoacetate $(2.0 \mathrm{mmol})$, hydrazine hydrate $(2.0 \mathrm{mmol})$, benzaldehyde $(2.0$ $\mathrm{mmol})$ and malononitrile $(2.0 \mathrm{mmol}) .{ }^{b}$ Isolated yield

Apart from the solvent, the efficiency of the multicomponent reactions is mainly affected by the amount of the catalyst and the reaction time. Thus, to enhance the yield of the desired product, we changed the amount of the catalyst from 5 to $20 \mathrm{~mol} \%$. As indicated in Table 3, maximum yield (94\%) was obtained when the reaction was loaded with $5 \mathrm{~mol} \%$ of the catalyst (regarding to the aldehyde).

Table 3. Influence of MTPB amount on the model reaction'

\begin{tabular}{cccc}
\hline Entry & MTPB, \% mol & Yield, $\%$ & Time, $\mathrm{h}$ \\
\hline 1 & 5 & 94 & 1 \\
2 & 10 & 82 & 1 \\
3 & 20 & 76 & 1 \\
\hline
\end{tabular}

With the optimized reaction conditions in hand and to study the efficiency of this catalyst, we extended our study with different aromatic aldehydes to prepare a series of pyranopyrazoles in good to excellent yields (Scheme 2, Table 4, 5a-m).

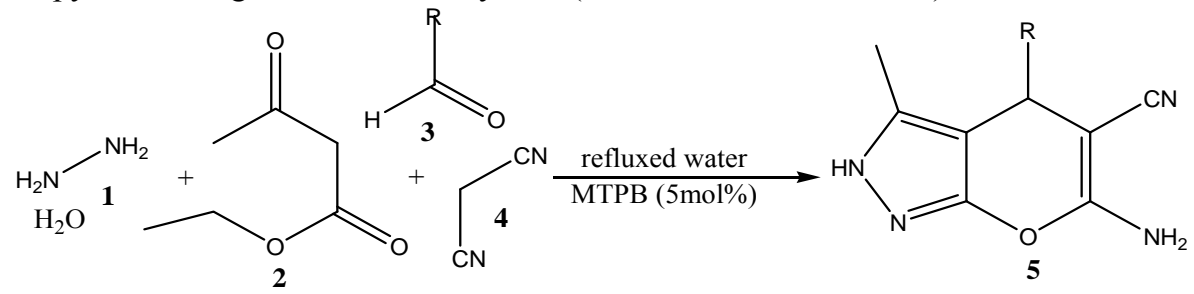

Scheme 2. Synthesis of pyranopyrazoles (5a-m)

Table 4. One-pot synthesis of pyranopyrazoles catalyzed by $\mathrm{MTPB}^{\mathrm{a}}$

\begin{tabular}{ccccccc}
\hline \multirow{2}{*}{ Entry } & \multirow{2}{*}{ Aldehyde } & \multirow{2}{*}{ Time, min } & \multirow{2}{*}{ Product } & \multirow{2}{*}{ Yield, \% } & \multicolumn{2}{c}{$\mathrm{mp},{ }^{\circ} \mathrm{C}$} \\
\hline 1 & $\mathrm{C}_{6} \mathrm{H}_{5}$ & 60 & $\mathbf{5 a}$ & 94 & $245-246$ & $243-245^{[9]}$ \\
2 & $4-\mathrm{Cl}-\mathrm{C}_{6} \mathrm{H}_{4}$ & 60 & $\mathbf{5 b}$ & 98 & $239-240$ & $233-235^{[9]}$ \\
3 & $4-\mathrm{OH}-\mathrm{C}_{6} \mathrm{H}_{4}$ & 60 & $\mathbf{5 c}$ & 93 & $229-230$ & $225-227^{[16]}$ \\
4 & $4-\mathrm{MeO}-\mathrm{C}_{6} \mathrm{H}_{4}$ & 90 & $\mathbf{5 d}$ & 89 & $225-226$ & $208-210^{[9]}$ \\
5 & $4-\mathrm{CH}_{3}-\mathrm{C}_{6} \mathrm{H}_{4}$ & 60 & $\mathbf{5 e}$ & 94 & $219-220$ & $206-207^{[16]}$ \\
6 & $4-\mathrm{NO}_{2}-\mathrm{C}_{6} \mathrm{H}_{4}$ & 60 & $\mathbf{5 f}$ & 93 & $248-250$ & $249-252^{[9]}$ \\
\hline
\end{tabular}




\begin{tabular}{ccccccc}
\hline 7 & $3-\mathrm{OH}-\mathrm{C}_{6} \mathrm{H}_{4}$ & 60 & $\mathbf{5 g}$ & 83 & $248-249$ & $248-249^{[16]}$ \\
8 & $3-\mathrm{NO}_{2}-\mathrm{C}_{6} \mathrm{H}_{4}$ & 120 & $\mathbf{5 h}$ & 85 & $215-217$ & $214-216^{[17]}$ \\
9 & $2-\mathrm{MeO}_{-}-\mathrm{C}_{6} \mathrm{H}_{4}$ & 90 & $\mathbf{5 i}$ & 95 & $253-254$ & $249-250^{[18]}$ \\
10 & $2-\mathrm{NO}_{2}-\mathrm{C}_{6} \mathrm{H}_{4}$ & 120 & $\mathbf{5 j}$ & 96 & $243-245$ & $243-244^{[18]}$ \\
11 & $4-\mathrm{Br}-\mathrm{C}_{6} \mathrm{H}_{4}$ & 60 & $\mathbf{5 k}$ & 90 & $180-183$ & $182-184^{[19]}$ \\
13 & Thien-2-yl & 60 & $\mathbf{5 l}$ & 94 & $226-228$ & $224-226^{[18]}$ \\
14 & $4-\mathrm{NMe}_{2}-\mathrm{C}_{6} \mathrm{H}_{4}$ & 60 & $\mathbf{5 m}$ & 80 & $219-222$ & $217-219^{[20]}$ \\
\hline
\end{tabular}

${ }^{a}$ Reactions were performed on a $2.0 \mathrm{mmol}$ scale of all reactants with $5 \mathrm{~mol} \%$ of MTPB in refluxed water $(5 \mathrm{~mL})$

On the basis of the chemistry of pyranopyrazoles, we propose the possible following mechanism: One molecule of hydrazine derivative $\mathbf{1}$ was firstly condensed with ethyl acetoacetate 2 to yield pyrazolone derivative 6. On the other hand, aromatic aldehyde 3 condensed with malononitrile 4 to afford $\alpha$-cyanocinnamonitrile derivative 7 . The next step may involve Michael addition of the active methylene of $\mathbf{6}$ to an electron deficient carbon of dicyanoalkene 7 , which gives an intermediate 8 tautomerization to the intermediate 9 followed by the nucleophilic attack of $\mathrm{OH}$ group on the cyano $(\mathrm{CN})$ moiety to give the cyclic intermediate 10, which is tautomerized to target pyranopyrazoles $\mathbf{5 a - m}$. In this process, MTPB could promote these reactions as phase transfer catalyst (Scheme 3).

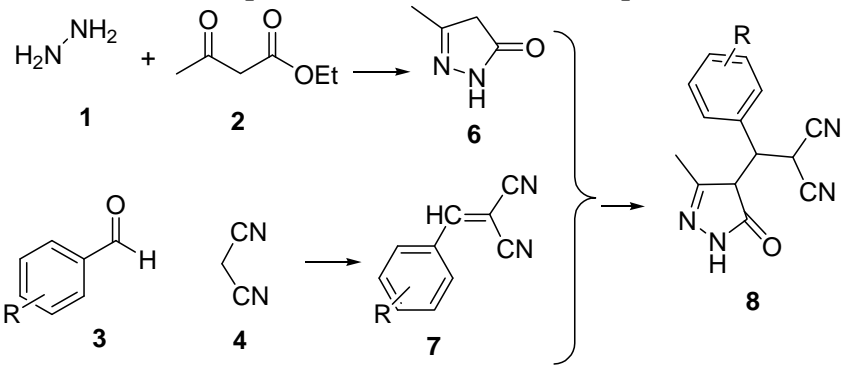<smiles>[R]c1cccc(C(c2c(C)n[nH]c2O)C(C#N)C(C#N)C(=O)O)c1</smiles><smiles>[R]c1ccc(C2C(C#N)=C(N)Oc3[nH]nc(C)c32)cc1</smiles><smiles>[R]c1ccccc1C1C(=N)Oc2[nH]nc(C)c2C1C#N</smiles>

Scheme 3. Plausible mechanism of pyranopyrazoles synthesis

\section{Conclusion}

The combination of multicomponent reactions (MCRs) and unconventional solvents has become a new research direction, which enables simultaneous growth of both MCRs and green solvents toward ideal organic synthesis. The present protocol couples the advantages of multi-component reactions (MCRs) with those of water as reaction media and ionic liquid (MTPB) as catalyst, thus representing a simple and green synthetic procedure to perform near-ideal methodologies for pyranopyrazoles synthesis.

\section{Acknowledgement}

We gratefully acknowledge the MESRES (Ministère de l'Enseignement Supérieur et de la Recherche Scientifique) for Financial support. 


\section{References}

1. (a) Ugi I, Pure Appl Chem., 2001, 73(1), 187-191;DOI:10.1351/pac200173010187

(b) Lie'by-Muller F, Simon C, Constantieux T and Rodriguez J, Comb Sci., 2006, 25, 432-438; (c) Evdokimov N M, Kireev A S, Yakovenko A A, Antipin M Y, Magedov I V and Kornienko A, J Org Chem., 2007, 72(9), 3443-3453; DOI:10.1021/jo070114u

2. Olivier-Bourbigou H and Magna L, J Mol Catal A: Chem., 2002, 419, 182-183; (b) Martins A P M, Clarissa P F, Dayse N M, Zanatta N and Bonacorso G H, Chem Rev., 2008, 108(6), 2015-2050; DOI:10.1021/cr078399y

3. (a) Anastas P T and Williamson T C, (Eds)., In Green Chemistry: Frontiers in Benign Chemical Syntheses and Processes,Oxford Press: New York, 1998, 1-50;

(b) DeSimone J M, Science, 2002, 297(5582), 799-803; DOI:10.1126/science.1069622

4. (a) Otto S and Engber J B F N, Pure Appl Chem., 2000, 72(7), 1365-1372; DOI:10.1351/pac200072071365 (b) Pirrung M C and Koushik Das Sarma, J Am Chem Soc., 2004, 126(2), 444-445; DOI:10.1021/ja038583a

5. (a) Kljin J E and Engberts J B F N, Nature, 2005, 435(7043), 746-747; DOI:10.1038/435746a (b) Narayan S, Fokin M, Kolb H and Sharpless K, Angew Chem Int Ed., 2005, 44(21), 3275-3279; DOI:10.1002/anie.200462883

6. Pirrung $\mathrm{M}$ and Das Sarma K, Tetrahedron, 2005, 61(48), 11456-11472; DOI:10.1016/j.tet.2005.08.068

7. Tacconi G, Gatti G, Desimoni G and Messori V A, J Prak Chem., 1980, 322, 831834; (b) El-Tamany E S, El-Shahed F A and Mohamed B H, J Serb Chem Soc., 1999, 64, 9-18; (c) Abdelrazek F M, Metz P, Metwally N H and El-Mahrouky S F, In Arch Pharm Chem Life Sci (Weinheim), 2006, 339(8), 456-460; DOI:10.1002/ardp.200600057 (d) Abdelrazek F M, Metz P, Kataeva O, Jäger A and EI-Mahrouky S F, In Arch Pharm Life Sci., 2007, 340(10), 543-548; DOI:10.1002/ardp.200700157

8. Junek $\mathrm{H}$ and Aigner H, Chem Ber., 1973, 106(3), 914-921; DOI:10.1002/cber.19731060323

9. Peng Y, Song G and Dou R, Green Chem., 2006, 8, 573-575; DOI:10.1039/B601209D

10. Sharanin Yu A, Sharanina L G and Puzanova V V, Zh Org Khim., 1983, 19, 2609-2615.

11. Vasuki G and Kandhasamy K, Tetrahedron Lett., 2008, 49(39), 5636-5638; DOI:10.1016/j.tetlet.2008.07.055

12. Madhusudana Reddy M B, Jayashankara V P and Pasha M A, Syn Commun., 2010, 40(19), 2930-2934; DOI:10.1080/00397910903340686

13. (a) Mecadon H, RohmanMd R, Rajbangshi M and Myrboh B, Tetrahedron Lett., 2011, 52(19), 2523-2525; DOI:10.1016/j.tetlet.2011.03.036 (b) Mecadon H, Rohman M R, Kharbangar I, Laloo B M, Kharkongor I, Rajbangshi M and Myrboh B, Tetrahedron Lett., 2011, 52(25), 3228-3231; DOI:10.1016/j.tetlet.2011.04.048

14. Kanagaraj K and Pitchumani K, Tetrahedron Lett., 2010, 51(25), 3312-3316; DOI:10.1016/j.tetlet.2010.04.087

15. Mingshu Wu, Qinqin Feng, Dehui Wan and Jinya Ma, Syn Commun., 2013, 43(12), 1721-1726; DOI:10.1080/00397911.2012.666315

16. Santhosh Reddy Mandha, Sravanthi Siliveri, Manjula Alla, Vittal Rao Bommena, Madhava Reddy Bommineni and Sridhar Balasubramanian, Bioorg Med Chem Lett., 2012, 22(16), 5272-5278; DOI:10.1016/j.bmcl.2012.06.055

17. Hemant V, Chavan, Santosh B Babar, Rahul U Hoval and Babasaheb P Bandgar, Bull Korean Chem Soc., 2011, 32(11), 3963; DOI:10.5012/bkcs.2011.32.11.3963 
18. Rui-Yun Guo, Zhi-Min An, Li-Ping Mo, Shu-Tao Yang, Hong-Xia Liu, Shu-Xia Wang and Zhan-Hui Zhang, Tetrahedron, 2013, 69(47), 9931-9938; DOI:10.1016/j.tet.2013.09.082

19. Niknam $\mathrm{K}$ and Piran A, Green Sustainable Chem., 2013, 3, 1-8; DOI:10.4236/gsc.2013.32A001

20. Kiyania H, Samimib H Allah, Ghorbania F and Esmaielia S, Current Chem Lett., 2013, 2, 197-206; DOI:10.5267/j.ccl.2013.07.002 